\title{
High vancomycin resistance among biofilms produced by Staphylococcus species isolated from central venous catheters
}

\author{
Ana Lúcia Souza Antunes ${ }^{1 /+}$, Jéssica Weis Bonfanti', Leandro Reus Rodrigues Perez', \\ Camille Cattani Ferreira Pinto', Ana Lúcia Peixoto de Freitas', \\ Alexandre José Macedo ${ }^{1,2}$, Afonso Luis Barth ${ }^{1,3}$ \\ ${ }^{1}$ Faculdade de Farmácia ${ }^{2}$ Centro de Biotecnologia ${ }^{3}$ Hospital de Clínicas de Porto Alegre, \\ Universidade Federal do Rio Grande do Sul, Av. Ipiranga 2752, 90610-000, Porto Alegre, RS, Brasil
}

\begin{abstract}
Biofilm production is an important mechanism that allows microbes to escape host defences and antimicrobial therapy. Vancomycin has been used largely for the treatment of methicillin-resistant staphylococcal infections. Here, we determined the minimal inhibitory concentration (MIC) and minimal biofilm eradication concentration (MBEC) for 82 Staphylococcus species isolated from central venous catheters $(\mathrm{CVC})$. Our results showed that the 41 strong and moderate-biofilm-producing isolates presented a higher MBEC/MIC ratio for vancomycin than the 24 weakbiofilm-producing isolates, illustrating the importance of biofilm production ability and the difficulty in treating biofilm-related infections. The MBEC was significantly higher in moderate-biofilm-producing isolates than in weakbiofilm-producing isolates $(p<0.001)$ and in strong-biofilm-producing isolates than in weak-biofilm-producing isolates $(p=0.001)$. The correlation between the MIC and the MBEC was poor. Based on our results, we recommend that bacterial biofilms be suspected in all cases of CVC infection.
\end{abstract}

Key words: biofilm - vancomycin - Staphylococcus spp

Staphylococcus spp is a major concern of the medical community, mainly in medical devices, such as central venous catheters (CVC) (LaPlante \& Mermel 2007). Vancomycin is the therapeutic choice for the treatment of methicillin-resistant staphylococcal infections, but over the last decade, isolates with reduced susceptibility to glycopeptides have emerged around the world (Tenover 2006). A methicillin-resistant Staphylococcus aureus (MRSA) isolate with reduced susceptibility to vancomycin, known as vancomycin-intermediate $S$. aureus (VISA), was first found in Japan in 1997 (Tenover 2006). The exact mechanisms by which VISA isolates become resistant to vancomycin remain unclear, but they probably involve thickening of the cell wall due to the accumulation of cell wall fragments capable of binding vancomycin extracellularly and changes in several metabolic pathways that slow cell growth (Tenover 2006). The vancomycin molecules are sequestered in the outer layers of the cell wall, thereby limiting access to the cytoplasmic membrane. A crucial step in the pathogenesis of these infections is the formation of a stable biofilm on the surface of the implanted biomedical device; biofilm formation is considered an important virulence factor for staphylococci (Stepanović et al. 2007).

Financial support: HCPA, CNPq, CAPES

+ Corresponding author: analucia2112@gmail.com

Received 25 May 2010

Accepted 20 September 2010
A biofilm is a structured community of bacterial cells that is enclosed in a self-produced polymeric matrix and adheres to an inert or living surface. Biofilm formation on implant surfaces increases the resistance to antimicrobial agents and leads to the therapeutic failure of conventional antimicrobial agents. Vancomycin diffuses slowly into the inner layers of bacterial biofilms; the gradual exposure of the bacterial cells to low concentrations facilitates the development of vancomycin resistance. It should be noted that even among the more susceptible isolates, subpopulations develop resistance and/or intermediate susceptibility to vancomycin after exposure (Rice 2006). The aim of this study was to evaluate the increase in vancomycin resistance among Staphylococcus species by comparing the minimal inhibitory concentration (MIC) and minimal biofilm eradication concentration (MBEC).

\section{SUBJECTS, MATERIALS AND METHODS}

Isolates - A total of 224 species of Staphylococcus were isolated from the CVCs of patients at the Clinics Hospital in Porto Alegre (HCPA), Rio Grande do Sul, a tertiary care hospital located in Southern Brazil, from January 2008-May 2009. Catheter cultures were made according to Maki et al. (1977).

Identification of the isolates - The isolates were cultured in agar supplemented with 5\% sheep blood (Tryptic Soy Agar-Oxoid, Basingstoke, UK) for $24 \mathrm{~h}$ at $35^{\circ} \mathrm{C}$ and their colony morphology and hemolysis were evaluated. Subsequently, coagulase and catalase tests were performed. The staphylococcal species that tested negative for coagulase were identified according to Antunes et al. (2008) and confirmed as non-epidermidis coagulasenegative staphylococci by the WalkAway ${ }^{\circledR}$ automated system (Siemens, USA). 
Criteria of inclusion - For this study, we choose to work with all strong-biofilm-producing isolates (n $=17)$, all moderate-biofilm-producing isolates $(\mathrm{n}=$ $24)$, weak-biofilm-producing isolates $(n=24)$ and 17 non-biofilm-producing isolates, for a total of 82 isolates evaluated. Due to the high rate of resistance to $\beta$-lactams, we choose to work with vancomycin, which is the first line for treatment of methicillin-resistant staphylococcal infections.

Ethics - Approval for the study was obtained from the National Committee for Ethics in Research of the HCPA ethics committee and from the Institutional Review Board of the Brazilian Office for Human Research Protection.

Antimicrobial susceptibility test - Disc diffusion testing was carried out as recommended by the Clinical and Laboratory Standards Institute (CLSI 2009). The following antimicrobial agents (Oxoid, Basingstoke, UK) were tested: $2 \mu \mathrm{g}$ clindamycin, $30 \mu \mathrm{g}$ chloramphenicol, $30 \mu \mathrm{g}$ doxycycline, $15 \mu \mathrm{g}$ erythromycin, $10 \mu \mathrm{g}$ gentamicin, $5 \mu \mathrm{g}$ levofloxacin, $1.25 / 23.75 \mu \mathrm{g}$ trimethoprim/sulphamethoxazole, $5 \mu \mathrm{g}$ oxacillin, $5 \mu \mathrm{g}$ rifampicin and $30 \mu \mathrm{g}$ vancomycin. The percentage of resistance was calculated with the overall resistance to all antimicrobial agents for each category of isolates (strong, moderate and weakbiofilm producing and non-biofilm producing).

MIC - The MIC for vancomycin (Sigma-Aldrich, St. Louis, MO, USA) was determined by microtitre plate assays as recommended by the CLSI guidelines (CLSI 2003). This procedure was performed in quadruplicate and the MICs were read manually after a 24-h incubation.

Biofilm formation assay - Biofilm formation assays and the characterization of the isolates were performed according to Stepanović et al. (2007). The isolates were cultured in Muller-Hinton agar (Oxoid, Basingstoke, UK) for $24 \mathrm{~h}$ at $35^{\circ} \mathrm{C}$ and then $20 \mu \mathrm{L}$ inocula of bacterial suspensions were prepared ( 0.5 McFarland). These inocula were added to each well of a sterile 96-well polystyrene flat-bottom microtitre plate (Costar 3599, Corning, NY, USA) and the wells were filled with $180 \mu \mathrm{L}$ of trypticase soy broth (TSB) medium (Oxoid; Basingstoke, UK) supplemented with $0.25 \%$ glucose for Staphylococcus epidermidis and $1 \%$ glucose for S. aureus, respectively. The plates were incubated for $18 \mathrm{~h}$ at $35^{\circ} \mathrm{C}$. To remove non-adherent cells, the wells were rinsed three times with sterile saline. The attached bacteria were fixed with methanol for $20 \mathrm{~min}$ and dried for $30 \mathrm{~min}$ at room temperature. Crystal violet $(0.5 \%)$ was used to stain the bacteria for $15 \mathrm{~min}$. The biofilm was eluted with ethanol for $30 \mathrm{~min}$ without shaking. The absorbance at $450 \mathrm{~nm}$ was measured in a microtitre plate reader (Behring EL 311, Hoechst, Akasaka, Japan). TSB was used as a spectrophotometric blank. The mean absorbance values and their standard deviations (SD) were calculated. The average optical density $\left(\mathrm{OD}_{450}\right)$ values were calculated for all tested strains and negative controls, performed in quadruplicate and repeated three times. The cut-off value $\left(\mathrm{OD}_{\mathrm{C}}\right)$ was defined as threefold the SD above the mean OD of the negative controls:
$\mathrm{OD}_{\mathrm{C}}=$ average $\mathrm{OD}$ of the negative controls $+(3 \mathrm{xD}$ of negative controls). The OD value of a tested strain was expressed as the average OD value of the strain minus the $\mathrm{OD}_{\mathrm{C}}$ value $\left(\mathrm{OD}=\right.$ average $\mathrm{OD}$ of strain $\left.-\mathrm{OD}_{\mathrm{C}}\right)$. The $\mathrm{OD}_{\mathrm{C}}$ value was calculated separately for each microtitre plate. The isolates were classified in accordance with Stepanović et al. (2007): non biofilm producer $=\mathrm{OD} \leq$ $\mathrm{OD}_{\mathrm{C}}$; weak biofilm producer $=\mathrm{OD}_{\mathrm{C}}<\mathrm{OD} \leq 2 \times \mathrm{OD}_{\mathrm{C}}$; moderate biofilm producer $=2 \times \mathrm{OD}_{\mathrm{C}}<\mathrm{OD} \leq 4 \times \mathrm{OD}_{\mathrm{C}}$; strong biofilm producer $=4 \times \mathrm{OD}_{\mathrm{C}}<\mathrm{OD}$.

$M B E C$ - The MBEC assay was performed as described by Moskowitz et al. (2004) with a few modifications to make the procedure more compatible with routine clinical microbiology laboratory practices. The isolates were cultured in Muller-Hinton agar (Oxoid, Basingstoke, England) for $24 \mathrm{~h}$ at $35^{\circ} \mathrm{C}$ and then $20 \mu \mathrm{L}$ of inocula of bacterial suspensions $\left(\sim 10^{8}\right.$ colonyforming unit $/ \mathrm{mL}$ ) were prepared. These inocula were added to each well of a sterile 96-well polystyrene flat-bottom microtitre plate that was then filled with $180 \mu \mathrm{L}$ per well of TSB medium supplemented with $0.25 \%$ glucose for $S$. epidermidis and $1 \%$ glucose for S. aureus. The plates were incubated for $18 \mathrm{~h}$ at $35^{\circ} \mathrm{C}$ and the non-adherent cells were removed by washing the wells with $200 \mu \mathrm{L}$ of sterile saline. The remaining attached bacteria was re-suspended in $100 \mu \mathrm{L}$ of cation adjusted Mueller-Hinton broth (Oxoid, Basingstoke, UK) and challenged with a solution of $100 \mu \mathrm{L}$ of vancomycin at different concentrations $(0.25,0.5$, $1.0,2.0,4.0,8.0,16.0,32.0$ and $64.0 \mu \mathrm{g} / \mathrm{mL}$ ). The plates were incubated for $24 \mathrm{~h}$ at $35^{\circ} \mathrm{C}$. The vancomycin was removed and the wells were rinsed three times with sterile saline. The subsequent steps (i.e., fixation, staining and elution) were performed as in the biofilm formation assay. The MBEC was defined as the minimum concentration of antibiotic required to eradicate the biofilm. Eradication of the biofilm gave an $\mathrm{OD}_{450}$ reading of approximately 0.080 , which is similar to the readings for the negative controls. All biofilm experiments were performed in quadruplicate for each isolate and repeated independently three times to minimize the variability in the OD measurements. Mean values and SDs were calculated. There was no variance among the results when performed in quadruplicate.

Quality control - The following reference isolates were used for quality control: S. epidermidis ATCC 35984 (biofilm producing), S. epidermidis ATCC 12224 (non-biofilm producing) for the microtitre plate and MBEC assays and S. aureus ATCC 25213 and S. aureus ATCC 29213 for the MIC assay.

Statistical analysis - The statistical significance of associations between variables in different categories of isolates (strong, moderate and weak-biofilm-producing $\mathrm{x}$ ratio MBEC/MIC) was calculated using the Kruskal-Wallis one-way analysis of variance test, which is an extension of the Mann-Whitney U test, following the test procedure proposed by Dunn. For all tests, differences were considered significant at $p<0.05$. All statistical analyses were performed using SPSS for Windows, release 18.0. 


\section{RESULTS}

Biofilm production was evaluated for a total of 224 isolates, including $185(82.6 \%)$ coagulase negative staphylococci (CNS) and 39 (17.4\%) S. aureus. Among these isolates, $118 / 185 \mathrm{CNS}$ were biofilm producers and $36 / 39 \mathrm{~S}$. aureus isolates were biofilm producers. For the purpose of the MBEC evaluation, we considered only some of the biofilm-producing isolates, including all the strong and moderate ones and as many distinct species of the weak and non-producing isolates as possible. As a result, 82 of the isolates could be identified; 20 were $S$. aureus, 55 were $S$. epidermidis, three were Staphylococcus haemolyticus, two were Staphylococcus capitis subsp. ureolyticus, one was $S$. capitis subsp. capitis and one was Staphylococcus sciuri (Table). These isolates were also challenged with 10 antimicrobials and the resistance rate for strong biofilm producers was 45\% (77/170), that for moderate biofilm producers was $45 \%$ (107/240), that for weak biofilm producers was 34\% (81/240) and that for non-biofilm producers was $28 \%$ (47/170). The vast majority (58/65) of biofilm-producing isolates presented high MBEC values ( $\geq 8 \mu \mathrm{g} / \mathrm{mL})$ and could be considered non-susceptible to vancomycin. A higher MBEC/MIC ratio of $\sim 64$ was found in six strong-biofilm-producing isolates (all $S$. epidermidis) and two moderate-biofilmproducing isolates (1 S. epidermidis and $1 \mathrm{~S}$. capitis subsp. capitis). We have noted that among weak-biofilmproducing isolates a higher MBEC/MIC ratio (equal to 16) was noted only for one $S$. sciuri isolate. All biofilmproducers presented MBECs that were higher than the MIC for vancomycin. The difference between the values of the MBEC and MIC was more evident for isolates with a moderate or strong capacity to form biofilms. The non-biofilm-producing isolates showed the same values

TABLE

Status of biofilm formation according to minimal inhibitory concentration (MIC) and minimal biofilm eradication concentration (MBEC) values among staphylococci species

\begin{tabular}{|c|c|c|c|c|c|}
\hline $\begin{array}{l}\text { Isolates } \\
(\mathrm{n}=82)\end{array}$ & Species & Category & $\begin{array}{c}\mathrm{MIC} \\
(\mu \mathrm{g} / \mathrm{mL})\end{array}$ & $\begin{array}{l}\text { MBEC } \\
(\mu \mathrm{g} / \mathrm{mL})\end{array}$ & $\begin{array}{c}\text { Ratio } \\
(\mathrm{MBEC} / \mathrm{MIC})^{a}\end{array}$ \\
\hline 1 & Staphylococcus aureus & Strong & 1 & 16 & 16 \\
\hline 4 & Staphylococcus epidermidis & Strong & 2 & 8 & 4 \\
\hline 2 & S. epidermidis & Strong & 2 & 16 & 8 \\
\hline 6 & S. epidermidis & Strong & 1 & $>64$ & $\sim 64$ \\
\hline 4 & S. epidermidis & Strong & 2 & $>64$ & $\sim 32$ \\
\hline 2 & S. aureus & Moderate & 0.5 & 16 & 32 \\
\hline 6 & S. epidermidis & Moderate & 1 & 8 & 8 \\
\hline 2 & S. epidermidis & Moderate & 2 & 8 & 4 \\
\hline 1 & S. epidermidis & Moderate & 1 & 16 & 16 \\
\hline 2 & S. epidermidis & Moderate & 1 & 32 & 32 \\
\hline 3 & S. epidermidis & Moderate & 1 & $>64$ & $\sim 64$ \\
\hline 6 & S. epidermidis & Moderate & 2 & $>64$ & $\sim 32$ \\
\hline 1 & Staphylococcus capitis ureolyticus & Moderate & 2 & $>64$ & $\sim 32$ \\
\hline 1 & S. capitis capitis & Moderate & 1 & $>64$ & $\sim 64$ \\
\hline 4 & S. aureus & Weak & 1 & 4 & 4 \\
\hline 6 & S. aureus & Weak & 1 & 8 & 8 \\
\hline 2 & S. epidermidis & Weak & 1 & 4 & 4 \\
\hline 4 & S. epidermidis & Weak & 1 & 8 & 8 \\
\hline 4 & S. epidermidis & Weak & 2 & 8 & 4 \\
\hline 1 & S. epidermidis & Weak & 2 & 16 & 8 \\
\hline 1 & Staphylococcus haemolyticus & Weak & 1 & 4 & 4 \\
\hline 1 & S. haemolyticus & Weak & 2 & 8 & 4 \\
\hline 1 & Staphylococcus sciuri & Weak & 1 & 16 & 16 \\
\hline 7 & S. aureus & Non producing & 1 & 1 & 1 \\
\hline 1 & S. haemolyticus & Non producing & 2 & 2 & 1 \\
\hline 6 & S. epidermidis & Non producing & 1 & 1 & 1 \\
\hline 2 & S. epidermidis & Non producing & 2 & 2 & 1 \\
\hline 1 & S. capitis ureolyticus & Non producing & 1 & 1 & 1 \\
\hline
\end{tabular}

$a: \mathrm{p}$ values gives the significance of differences in MBEC/MIC between the categories in two groups: (i) strong $\mathrm{x}$ weak ( $\mathrm{p}=$ $0.001)$ and moderate $\mathrm{x}$ weak $(\mathrm{p}<0.001)$ and (ii) no significance between strong $\mathrm{x}$ moderate $(\mathrm{p}=1)$. 
for the MIC and MBEC. The MBEC was significantly higher in moderate-biofilm-producing isolates than in weak-biofilm-producing isolates $(p<0.001)$ and in strong-biofilm-producing isolates than weak-biofilmproducing isolates $(\mathrm{p}=0.001)$. However, when we compared the MBECs of strong-biofilm-producing isolates with those of moderate-biofilm-producing isolates, the differences were not significant (Table).

\section{DISCUSSION}

Vancomycin is still used intensely for the treatment of MRSA bacteremia, as well as other methicillin-resistant staphylococcal infections. However, vancomycin treatment sometimes fails, mainly when the isolates are borderline susceptible to vancomycin (Musta et al. 2009, Rybak et al. 2009). The failure of vancomycin to treat MRSA infections has been widely reported (Graninger et al. 2002). Additionally, biofilm formation is largely associated with infections due to contamination medical devices, such as CVCs. In these cases, eradication of the infection is difficult because the antimicrobial agents may not penetrate inside the biofilm (Stewart 2002). This condition is attributed to a possible barrier function of the biofilm binding the antimicrobial agents within the matrix. Jefferson et al. (2005) have demonstrated that vancomycin partially permeated a $S$. aureus biofilm during $1 \mathrm{~h}$ of exposure to the drug under static conditions. Hospitalization and hemodialysis are important risk factors for the development of bloodstream catheter-related infection caused by S. epidermidis and $S$. aureus. Systemic antimicrobial therapy should be initiated as a prophylactic measure against infection (LaPlante \& Mermel 2007, Raad et al. 2007). Despite the large number of antibacterial agents available, none have been found to totally eradicate bacterial biofilms. Various studies have shown that an antimicrobial agent used for disruption of the biofilm matrix can facilitate the penetration of another antimicrobial agent into the biofilm (Glansdorp et al. 2008, Hajdu et al. 2009, Presterl et al. 2009). The resistance of biofilms can mostly be attributed the decreased diffusion of antimicrobial agents through the extensive biofilm matrix (Rice 2006, Tenover 2006, Frank et al. 2007) as well as the decreased metabolic activity of bacteria within biofilms and the increase in gene transfer (Amorena et al. 1999).

There was no significant correlation between the biofilm phenotype and the hospital unit, previous use of antibiotics, time of catheter removal, length of stay in the hospital, associated infections, predisposing conditions, outcome, age or gender. These observations might be due to classification of the institution as a tertiary-care teaching hospital that receives a heterogeneous group of patients, particularly the critically ill. The only correlation found was that the biofilm-producing isolates presented higher rates of resistance to some antibiotics used in therapy compared to the non-producing biofilm isolates.

This study evaluated the antimicrobial susceptibility profile of biofilms and planktonic cells of a set of staphylococcal isolates. Our results demonstrated that the vancomycin resistance is higher in the biofilm mode of growth than in the planktonic mode of growth. Among strong and moderate-biofilm-producing isolates, a higher MBEC/MIC ratio ( 64 for the 6 strong-biofilm-producing S. epidermidis and the 4 moderate-biofilm-producing isolates, 3 of which were S. epidermidis and 1 of which was $S$. capitis subsp capitis) was observed. Among the weak biofilm producers, only one $S$. sciuri isolate showed a MBEC/MIC ratio of 16. Strong and moderate-biofilm-producing $S$. aureus isolates (1 and 2 isolates, respectively) showed higher MBEC/MIC ratios than weak-biofilm-producing $S$. aureus (10 isolates), indicating decreased susceptibility. Among the biofilm-forming isolates, $89 \%$ showed high vancomycin MBEC $(\geq 8 \mu \mathrm{g} / \mathrm{mL})$ and cannot be considered susceptible to vancomycin according to the CLSI breakpoints (CLSI 2009). All these isolates, however, presented vancomycin MICs of $\leq 2 \mu \mathrm{g} / \mathrm{mL}$, which is within the "susceptible" category. This observation shows that biofilm production results in an important barrier to antimicrobial diffusion into the biofilm. Among S. epidermidis isolates $(\mathrm{n}=55)$ high $\mathrm{MBEC} /$ MIC ratios were observed because theses isolates were strong (16 isolates) and moderate (20 isolates) producers of biofilms. All the non-biofilm-producing isolates presented low MBECs $(\leq 2 \mu \mathrm{g} / \mathrm{mL})$ and were susceptible to vancomycin. $S$. epidermidis is the agent most associated with CVC infections. Its ability to produce biofilms explains, in part, its high prevalence in this type of infection.

We observed a significant increase in the MBEC/ MIC ratio among strong and moderate-biofilm-producing isolates when compared with weak-biofilm-producing isolates. Perhaps this finding can be explained by the slow diffusion of vancomycin into the deeper layers of bacterial biofilms, which may promote resistance due to the gradual exposure of the bacterial cells to low concentrations of the antibiotic.

Recent studies have shown the importance of the accessory gene regulators, a global quorum-sensing complex regulator in $S$. aureus that is responsible for orchestrating the expression of adherence factors, biofilm production, tolerance to vancomycin and many other virulence factors. Additionally, the accessory gene regulators locus has been studied intensely because it appears to be associated with a genetic polymorphism in this cluster and patient response to vancomycin therapy (Sakoulas et al. 2005, Monaco et al. 2010).

In conclusion, choosing the correct antimicrobial therapy for the treatment of biofilm-related infections appears to require the application of specific biofilm assays; antimicrobial susceptibility testing based on MIC values alone cannot accurately determine the exact susceptibility of bacterial biofilms. In this paper we draw attention to the poor correlation between the MIC and the MBEC. In some cases, such as CVC infections, it is crucially important to suspect bacterial biofilm production. The sensitivity of planktonic cells to vancomycin has been closely monitored because of the emergence of staphylococcal species with reduced susceptibility. For these traits, the ability to produce biofilms would enhance their capacity to acquire antibiotic resistance and to respond to adverse conditions. 


\section{REFERENCES}

Amorena B, Gracia E, Monzón M, Leiva J, Oteiza C, Pérez M, Alabart JL, Hernández-Yago J 1999. Antibiotic susceptibility assay for Staphylococcus aureus in biofilms developed in vitro. $J$ Antimicrob Chemother 44: 43-55.

Antunes AL, Secchi C, Reiter KC, Perez LR, de Freitas AL, D'Azevedo PA 2008. Feasible identification of Staphylococcus epidermidis using desferrioxamine and fosfomycin disks. APMIS 116: $16-20$.

CLSI - Clinical and Laboratory Standards Institute 2003. Methods for dilution antimicrobial susceptibility tests for bacteria that grow aerobically: approved Standard, NCCLS document M7-A6, 6th ed., CLSI/NCCLS, Wayne, 1-25.

CLSI - Clinical and Laboratory Standards Institute 2009. Performance standards for antimicrobial disk susceptibility testing, 19th informational supplement, CLSI, Wayne, $10 \mathrm{pp}$.

Frank KL, Reichert EJ, Piper KE, Patel R 2007. In vitro effects of antimicrobial agents on planktonic and biofilm forms on Staphylococcus lugdunensis clinical isolates. Antimicrob Agents Chemother 51: 888-895.

Glansdorp FG, Spandl RJ, Swatton JE, Loiseleur O, Welch M, Spring DR 2008. Using chemical probes to investigate the sub-inhibitory effects of azythromycin. Org Biomol Chem 6: 4120-4124.

Graninger W, Assadian O, Lagler H, Ramharter M 2002. The role of glycopeptides in the treatment of intravascular catheter-related infections. Clin Microbiol Infect 8: 310-315.

Hajdu S, Lassnigg A, Graninger W, Hirschl AM, Presterl E 2009. Effects of vancomycin, daptomycin, fosfomycin, tigecycline and ceftriaxone on Staphylococcus epidermidis biofilms. J Orthop Res 27: 1361-1365.

Jefferson KK, Goldmann DA, Pier GB 2005. Use of confocal microscopy to analyze the rate of vancomycin penetration through Staphylococcus aureus biofilms. Antimicrob Agents Chemother 49: 2467-2473.

LaPlante KL, Mermel LA 2007. In vitro activity of daptomycin and vancomycin lock solutions on staphylococcal biofilms in a central venous catheter model. Nephrol Dial Transplant 22: 2239-2246.

Maki DG, Jarrett F, Sarafin HW 1977. A semiquantitative culture method for identification of catheter-related infection in the burn patient. J Surg Res 22: 513-520.

Monaco M, Sanchini A, Grundmann H, Pantosti A 2010. Vancomycinheteroresistant phenotype in invasive methicillin-resistant
Staphylococcus aureus isolates belonging to spa type 041. Eur J Clin Microbiol Infect Dis 29: 771-777.

Moskowitz SM, Foster JM, Emerson J, Burns JL 2004. Clinically feasible biofilm susceptibility assay for isolates of Pseudomonas aeruginosa from patients with cystic fibrosis. J Clin Microbiol 42: $1915-1922$

Musta AC, Riederer K, Shemes S, Chase P, Jose J, Johnson LB, Khatib R 2009. Vancomycin MIC plus heteroresistance and outcome of methicillin-resistant Staphylococcus aureus bacteremia: trends over 11 years. J Clin Microbiol 47: 1640-1644.

Presterl E, Hajdu S, Lassnigg AM, Hirschl AM, Holinka J, Graninger W 2009. Effects of azithromycin in combination with vancomycin, daptomycin, fosfomycin, tigecycline and ceftriaxone on Staphylococcus epidermidis biofilms. Antimicrob Agents Chemother 53: 3205-3210.

Raad I, Hanna H, Jiang Y, Dvorak T, Reitzel R, Chaiban G, Sherertz R, Hachem R 2007. Comparative activities of daptomycin, linezolid and tigecycline against catheter-related methicillin-resistant Staphylococcus bacteremic isolates embedded in biofilm. Antimicrob Agents Chemother 51: 1656-60.

Rice LB 2006. Antimicrobial resistance in gram-positive bacteria. Am J Med 119 (Suppl. 1): S11-S19.

Rybak M, Lomaestro B, Rotschafer JC, Moellering R Jr, Craig W, Billeter M, Dalovisio JR, Levine DP 2009. Therapeutic monitoring of vancomycin in adult patients: a consensus review of the American Society of Health-System Pharmacists, the Infectious Diseases Society of America, and the Society of Infectious Diseases Pharmacists. Am J Health-Syst Pharm 66: 82-98.

Sakoulas G, Eliopoulos GM, Fowler VG Jr, Moellering RC Jr, Novick RP, Lucindo N, Yeaman MR, Bayer AS 2005. Reduced susceptibility of Staphylococcus aureus to vancomycin and platelet microbicidal protein correlates with defective autolysis and loss of accessory gene regulator (agr) function. Antimicrob Agents Chemother 49: 2687-2692.

Stepanović S, Vuković D, Hola V, Di Bonaventura G, Djukić S, Cirković I, Ruzicka F 2007. Quantification of biofilm in microtiter plates: overview of testing conditions and practical recommendations for assessment of biofilm production by staphylococci. APMIS 115: 891-899.

Stewart PS 2002. Mechanisms of antibiotic resistance in bacterial biofilms. Int J Med Microbiol 292: 107-113.

Tenover FC 2006. Mechanisms of antimicrobial resistance in bacteria. Am J Med 119 (Suppl. 1): S3-S10. 